\title{
Fluorescent variant of silica nanoparticle powder synthesised from rice husk for latent fingerprint development
}

\author{
Revathi Rajan ${ }^{1,2}$, Yusmazura Zakaria ${ }^{3}$, Shaharum Shamsuddin ${ }^{3}$ and Nik Fakhuruddin Nik Hassan ${ }^{2 *}$ (D)
}

\begin{abstract}
Background: Fingerprint, though still remains one of the best possible means of individual identification, is often lost during the recovery process. The successful utilisation of developed fingerprint for identification relies on the contrast between the ridges and substrate which allows for the minutiae recognition and characterisation. However, the contrast is difficult to be achieved on challenging or difficult surfaces such as reflective, multicoloured and patterned materials.

Methodology: Silica nanoparticles (SNP) were synthesised from rice husk using thermochemical treatment and incorporated with natural dyes. The photoluminescence of the powders was recorded and characterised using field emission scanning electron microscopy, Fourier-transform infrared spectroscopy, X-ray diffraction analysis and forensic alternative light source. The effectiveness of three fluorescent variant of the SNP powders and commercial fluorescent powder (CFP) was investigated by developing latent fingerprints deposited on different multicoloured substrates.
\end{abstract}

Results: Spherical fluorescent silica nanoparticles were successfully synthesised from rice husk. Characterisation studies of coloured silica nanoparticles showed amorphous spherical silica nanoparticle with a mean particle size of approximately $200 \mathrm{~nm}$. Silica nanoparticle doped with curcumin pigment exhibited strongest fluorescence and good stability when exposed to heat and aged at room temperature. The efficiency of the coloured silica nanoparticle was not compromised by the dye doping process as determined using electron microscope and surface tests on various surfaces.

Conclusion: Findings demonstrated that fluorescent silica powder synthesised from rice husk and curcumin dye extract exhibited good photoluminescence and hence providing sufficient contrast for the fingerprint analysis on difficult and challenging surfaces. Additionally, the spherical mono-dispersed nanoparticles enhanced the clarity and selectivity of the powder.

Keywords: Fluorescent silica nanoparticle, Natural pigments, Turmeric, Fingerprint, Patterned surface, Curcumin

\section{Background}

Fingerprints are the primary method of personal identification in a criminal investigation (Lennard 2014). Nevertheless, fingerprints that are recovered at crime scene normally require certain development techniques to acquire visibility to the naked eye (Jasuja et al. 2015). Recovering fingerprints on multicoloured, patterned or

\footnotetext{
* Correspondence: nikf@usm.my

${ }^{2}$ Forensic Science Programme, School of Health Sciences, Universiti Sains

Malaysia, 16150 Kubang Kerian, Kelantan, Malaysia

Full list of author information is available at the end of the article
}

otherwise luminescent surfaces remains challenging due to the lack of contrast observed in fingerprints developed using traditional powders (Becue et al. 2009). Black, grey or white powders that are commonly used for fingerprint dusting do not possess luminescent properties (Lee and Gaensslen 2012). Various synthetic luminescent fingerprint dusting powders are commercially available and proposed in many research articles (SIRCHIE 2012; Low et al. 2015; Errington et al. 2016).

Nano-technological inventions and innovations have exhibited significant impact in steering the fingerprint 
developing techniques towards enhanced sensitivity and selectivity. Targeted development of fingerprint ridges, luminescent fingerprints and chemical probing are amongst the enhancements that can be achieved using nanoparticles (Zhao et al. 2018; Li et al. 2018). The nanoparticles exploited in fingerprint research include gold, silver, titanium dioxide, zinc oxide and silica nanoparticles (SNPs) (Becue et al. 2007; Sodhi and Kaur 2017; Bergeron 2003; Becue et al. 2008; Kim et al. 2016; Newland et al. 2016; Choi et al. 2007; Choi et al. 2008; Singh et al. 2013).

Rare earth ion-doped materials have garnered recent interest in the field of forensic fingerprint development. Spherical-shaped crystalline calcium molybdate nanophosphors was proposed to exhibit high contrast and low background interference on several tested surfaces. The particle size of the fabricated nanophosphors varied in the range of 300 to $500 \mathrm{~nm}$ and emitted a red green fluorescence under ultraviolet (UV) light (Bharat et al. 2017). Utilising nanophosphors with persistent fluorescence for a period of time after irradiation with UV light alleviated the contrast issue resulted by high background fluorescence (Wang et al. 2017). Various other nanoparticles doped with fluorescent molecules to enhance contrast have been proposed in the past (Guzman et al. 2018; Prabakaran and Pillay 2019). Apart from using highly synthetic compound for latent fingerprint visualisation, the use of natural products for similar purpose was studied in current years. In other instances, natural fluorescent dye variants such as turmeric powder (Garg et al. 2011; Gaskell et al. 2013) and anthocyanin (Dorbut and Benchawattananon 2016) were applied for contrast enhancement.

Rice husk (RH), an agricultural waste that produced voluminously in all rice-producing countries, consists of few main components namely cellulose, lignin and silica (Chen et al. 2013). Copious extraction techniques of SNP from rice husk with varying degrees of purity and crystallinity were reported extensively (Mor et al. 2017; Vaibhav et al. 2015; Carmona et al. 2013). Techniques such as heat treatments (Lu and Hsieh 2012), thermochemical treatments (Wang et al. 2012; Ghorbani et al. 2015; Abu et al. 2016) and alkaline extraction followed by acidic precipitation (Hessien et al. 2009; Adam et al. 2011; Noushad et al. 2014) have been attempted, whereby most techniques produced impure silica, highly agglomerated and nanostructured SNPs (Li and Zhu 2011). Recently, spherical mono-dispersed SNP synthesised from rice husk was demonstrated to have excellent efficiency in developing latent fingerprints on non-porous and semi-porous surfaces (Rajan et al. 2016). In this study, we have explored for the first time the use of natural dye pigment doped spherical SNP synthesised from rice husk to enhance the contrast between fingerprint ridges and underlying substrates.

\section{Methods}

The SNP was synthesised from rice husk using thermochemical treatment. Highly pure rice husk ash was produced, then followed by alkaline solubilisation and acidic extraction. RH was soaked for an hour and rinsed with tap water to remove dirt and soil from milling. $\mathrm{RH}$ was transferred into porcelain crucibles and subjected to heat on hotplate until RH was semi charred. Semi charred $\mathrm{RH}$ was subjected to acid treatment. Charred husk was heated at $700{ }^{\circ} \mathrm{C}$ for $5 \mathrm{~h}$ in furnace until white silica ash was obtained. Sodium silicate was prepared by dissolving $\mathrm{RH}$ ash in $\mathrm{NaOH}$ under continuous stirring at $80^{\circ} \mathrm{C}$ for $1 \mathrm{~h}$. Sodium silicate was filtered, solid impurities removed and aged in the hot air oven at $80{ }^{\circ} \mathrm{C}$ overnight. Precipitation of silica nanoparticles was carried out using acetic acid and acetone (Rajan et al. 2016). White spherical mono-dispersed SNP ( $200 \mathrm{~nm})$ was obtained after freeze-drying process. Extraction of natural pigments was conducted using ethanol to facilitate the process through complete immersion of organic matter in the solvent. Curcumin, blue and purple anthocyanin and carotene pigments were extracted from turmeric, blue pea flower, purple cabbage and dried red chillies, respectively. Dried red chillies were subjected to digestion using sodium hydroxide to remove piquancy prior to pigment extraction (Chen and $\mathrm{Wu}$ 2009). All the extracted pigments were concentrated using rotary evaporator. Three synthetic dyes (safranin O, FCF brilliant blue food dye, Ponceau 4R red food dye) were also selected for the dye doping of the SNP. Characteristic pigment absorbance value was determined using Varian Cary 100 Bio Ultraviolet/Visible Spectrophotometer (Varian, Australia Ptd. Ltd.). Photoluminescence characteristic of the dyes was recorded under high intensity of ultraviolet light (350-380 nm).

Coloured SNP powder was produced by suspending the synthesised white SNP in ethanol until a homogenous colloid was formed. Concentrated pigment and pre-determined amount corn starch powder was added to the suspension and vigorously stirred to ensure homogenous dispersal of pigments. The solvent was evaporated to dryness at room temperature, leaving the coloured SNP. Corn starch was added to the mixture to increase the colour intensity of the powder. Different ratios of SNP powder to corn starch (from 10 to $40 \%$ ) were tested prior to the determination of optimal proportion to maintain efficacy of the original SNP powder and confer uniform colour distribution. Different ratios of powders were then applied on fresh fingerprints deposited on a clean glass slide. The powders were kept in dark bottle and stored in dry condition.

The morphological structure of the coloured SNP powder was analysed using Quanta FEG 450 field emission scanning electron microscope (FESEM), FEI 
Czech Republic. The prepared sample were spread on carbon tape mounted on an aluminium stubs. The stub mounted samples were sputter coated with gold under vacuum at $20 \mathrm{~mA}$ for $2 \mathrm{~min}$. The samples were observed and imaged at a 9-10 $\mathrm{mm}$ working distance and a 5.00 $\mathrm{kV}$ accelerating voltage. The surface chemical bonding and the functional groups were determined using Tensor 27 Fourier-transform infrared spectrophotometer (FTIR) by Bruker Corporations, UK. Samples were analysed as potassium bromide pellets and infrared spectra were collected within the range of 4000 to $400 \mathrm{~cm}^{-1}$ region in the transmittance mode.

The efficacy of three fluorescent variant of the SNP powders and commercial fluorescent powder (CFP) was determined by developing latent fingerprints on different multicoloured substrates namely two multicoloured metal cans, glossy wallpaper, borosilicate measuring cylinder, plastic multicoloured bottle, patterned porcelain mug, multicoloured plastic bag, multicoloured paper box, patterned leather and fluorescent paper. These substrates were selected because they pose greater challenge in obtaining appropriate contrast between the developed ridges and the background. However, the selected substrates were limited to smooth and non/semiporous surfaces as powder dusting technique only works best on such materials.

Four donors comprised of two adult males and two females (aged 20-50 years old) participated in this study. Individuals who have scars on fingers were excluded. The numbers of donors were in accordance with guidelines as recommended for a phase 1 project (three to five different donors for proof-of-concept investigation of novel fingerprint detection methods) by the International Fingerprint Research Group (IFRG) (2014). The guidelines however are not meant to be prescriptive as single donor is acceptable in this type of study (Azman et al. 2018). The protocol of this study was approved by The Human Research Ethics Committee of USM and informed consent from the donors was obtained. The donors were instructed to touch forehead and nose areas to mimic the natural action of touching such areas. One set of four groomed full fingerprints (right thumbprints) were gently deposited on each surface and then left at room temperature for one hour. The powders were applied using individual SIRCHIE squirrel hair brush using the powder dusting technique. Each fingerprint was photographed using forensic alternative light source (FALS) under appropriate filters. This study was conducted in May 2017.

\section{Results and discussion}

In comparison to the nanostructured powders available in the commercial fingerprint dusting powder, the spherical mono-dispersed SNP exhibited uniform and selective adhesion to fingerprint ridge residue (Rajan et al. 2016). The synthesised SNP had a mean particle size of $200 \mathrm{~nm}$. Nonetheless, the application of SNP powder is limited to dark backgrounds in order to produce sufficient contrast.

In this study, natural pigments present in plants and vegetables were extracted using alcohol. Anthocyanins are water soluble pigments, while the curcumin and carotene are not soluble in water. Anthocyanins exhibited thermal degradation when exposed to heat source for a prolonged period. Curcumin and carotene pigments demonstrated thermal stability even when exposed to temperature as high as $90{ }^{\circ} \mathrm{C}$. Hence, solvent evaporation can be accelerated using heat. The only drawback using carotene pigment extracted from dried red chillies was the pungent odour and sticky texture of the extract. As a result, the texture of the SNP powder produced using carotene pigment appeared clumping and possessed strong piquant smell. UV/Visible spectroscopic analysis was carried out to determine the maximum absorption wavelength $\left(\lambda_{\max }\right)$ of the dyes. Figure 1 shows characteristic absorption spectra for curcumin, anthocyanin purple, anthocyanin blue and carotenoids which exhibited at 423, 551, 573/617 and $439 \mathrm{~nm}$, respectively. On the other hand, the synthetic dyes safranin O, FCF brilliant blue and Ponceau 4R red displayed characteristic maximum absorptions at $513 \mathrm{~nm}, 620 / 407$ $\mathrm{nm}$ and $507 \mathrm{~nm}$ in agreement with previous reports (Balaji et al. 2011; Narayana and Divya 2010; Cheriaa et al. 2012; Bogner et al. 2011; Altınöz and Toptan 2002). Curcumin exhibited strongest fluorescence under high intensity of light source, in comparison to carotenoid and anthocyanins, while Safranin $O$ had the strongest fluorescence amongst the synthetic dyes (Fig. 2).

Uneven pigment distribution was observed in the coloured SNP produced without the addition of starch. This was presumably due to lack of pigment adherence directly to the surface of the SNP, which was then greatly improved by adding starch as a pigment binding agent. Starch was reported to work in conjunction with the anionic polymer to form hydrogen bonds and thereby provided optimal pigment dispersion (Patel et al. 2012). The ratio of starch to SNP was thoroughly investigated as not to compromise the clarity of the fingerprint developed using the spherical nanoparticle while conferring luminescent property to the particles. Figure 3 shows the fresh fingerprints developed using different ratios of SNP to starch. It was evident that 9:1 ratio preserved selectivity that also retained sufficient contrast. Curcumin has showed greater stability in terms of shelf life as compared to the other pigments.

Curcumin-doped powder had the strongest photoluminescence in comparison to the other powders. Powders doped with Safranin $\mathrm{O}$ and Ponceau 4R showed 

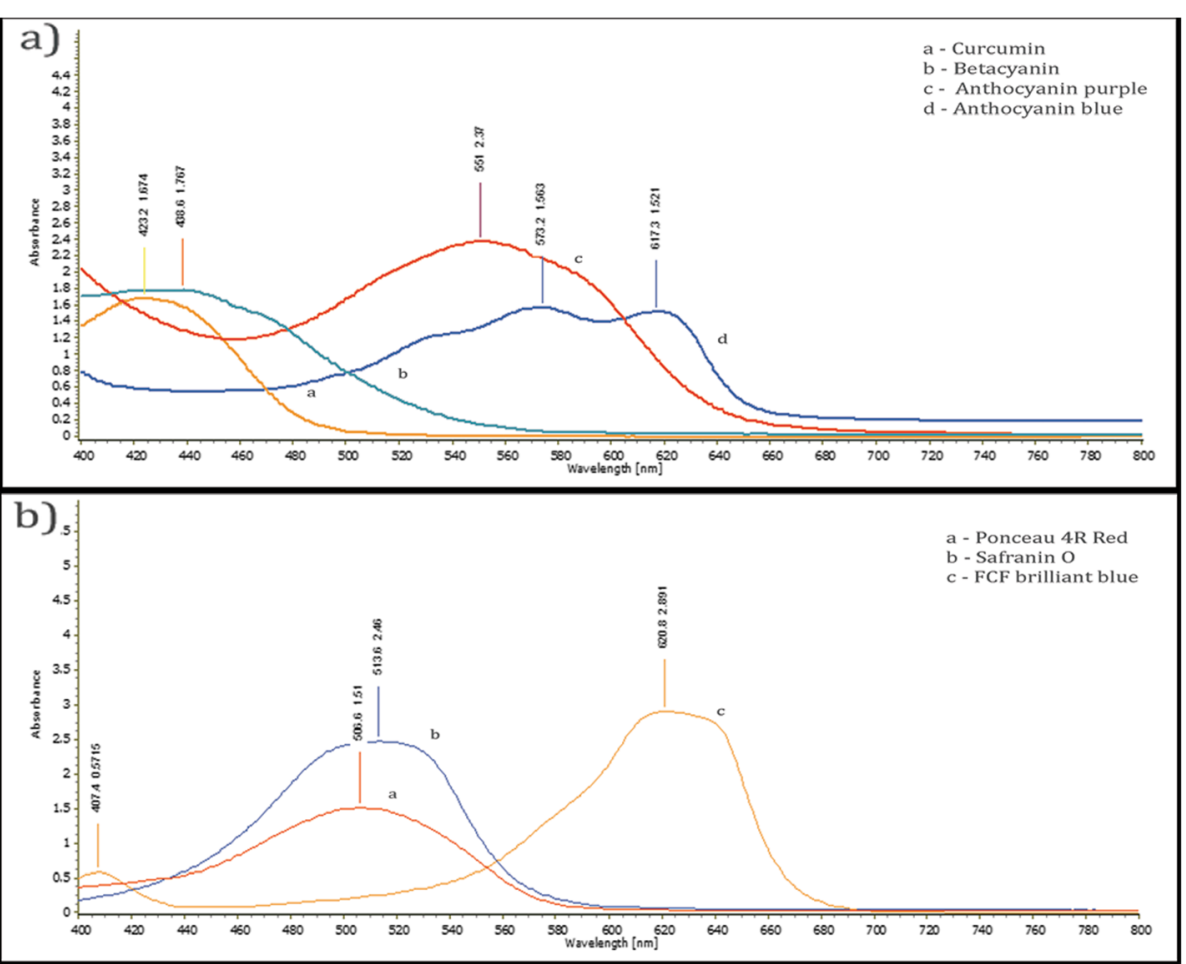

Fig. 1 UV-Visible absorbance spectra of a natural and $\mathbf{b}$ synthetic dyes

weak photoluminescence under high intensity of UV light. In addition, pigment degradation was observed in the powders doped with anthocyanins and carotenoids, while curcumin-doped powder remained stable for more than a year of shelf life. Three powders, which exhibited photoluminescence, were further characterised using FESEM and FTIR spectroscopy. Figure 4 depicts the coloured powder observed under white and UV light, as well as the fingerprints developed using these powders viewed under FALS. Photoluminescence study under UV light showed good luminescence intensity of the powders except for white SNP powder. However, the intensity significantly decreased when the powder was applied to fingerprint. Only curcumindoped SNP powder retained good contrast after its application to the fingerprints.

\section{Fluorescent SNP powder variants characterization}

Morphological analysis of the powders, shown in Fig. 5a$\mathrm{d}$, illustrates no significant difference between white and coloured SNP. Both observation signified that the efficacy of the original white powder, in terms of selectivity and

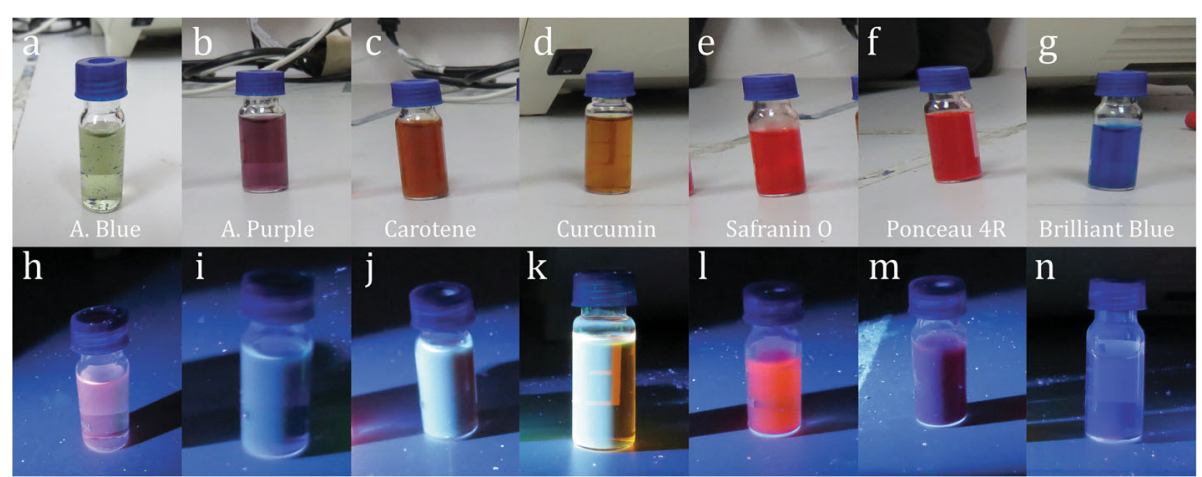

Fig. 2 Photoluminescence of dye pigments under different light sources $\mathbf{a}-\mathbf{g}$ white light and $\mathbf{h}-\mathbf{n}$ UV light 


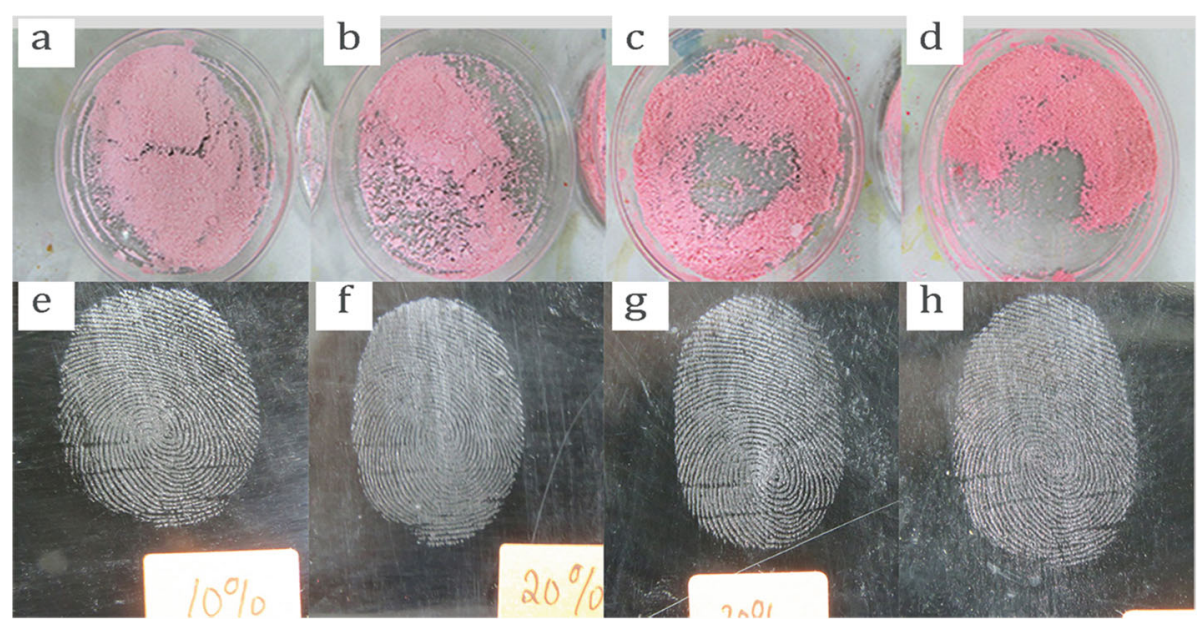

Fig. $\mathbf{3}$ Fingerprints developed (e-h) with pink SNP powders formulated with different amount of starch a 9 to 1 , b 8 to 2 , c 7 to 3 and $\mathbf{d} 6$ to 4 parts of SNP powder to starch ratio

sensitivity, remained as previously reported (Rajan et al. 2016). Spherical, mono-dispersed SNP with a mean particle size of $200 \mathrm{~nm}$ were observed in these micrographs of white and coloured powders. The morphology of the commercial luminescent yellow powder is depicted in Fig. 5e, whereby large blocks of several micrometre-sized particles can be observed. It has been emphasised in several literature that an effective fingerprint dusting powder should have characteristic such as small-sized and spherical, in order to elucidate a better resolution of the developed fingerprint (Sodhi and Kaur 2008; Moret et al. 2014). Figure 6

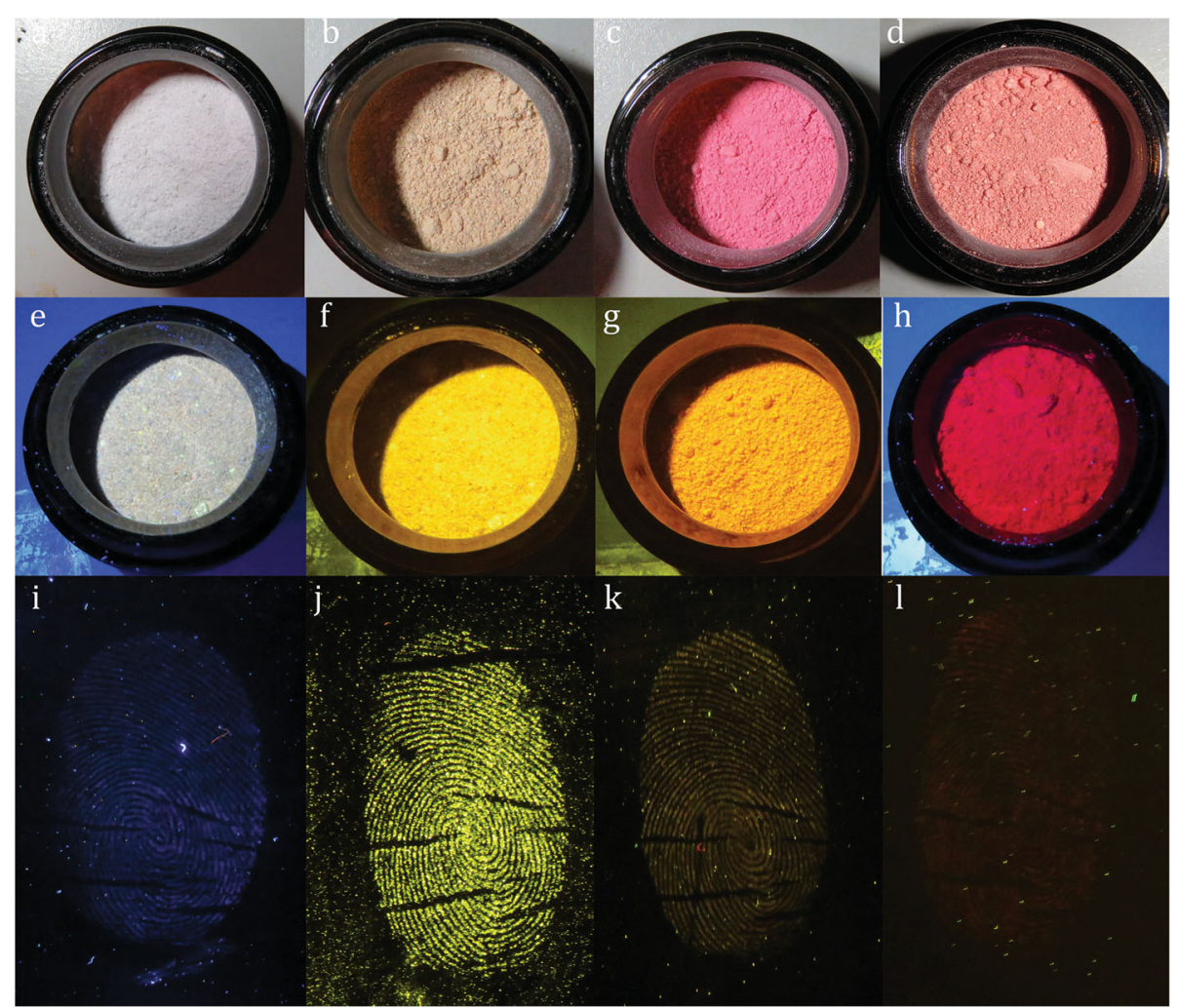

Fig. 4 White and fluorescent variants of SNP powders under white light a white $\mathbf{b}$ yellow $\mathbf{c}$ pink $\mathbf{d}$ red and viewed under blue light (420-470 nm) and orange filter $\mathbf{e}$ white $\mathbf{f}$ yellow $\mathbf{g}$ pink $\mathbf{h}$ red and fingerprints developed using $\mathbf{i}$ white $\mathbf{j}$ yellow $\mathbf{k}$ pink $\mathbf{I}$ red powders 


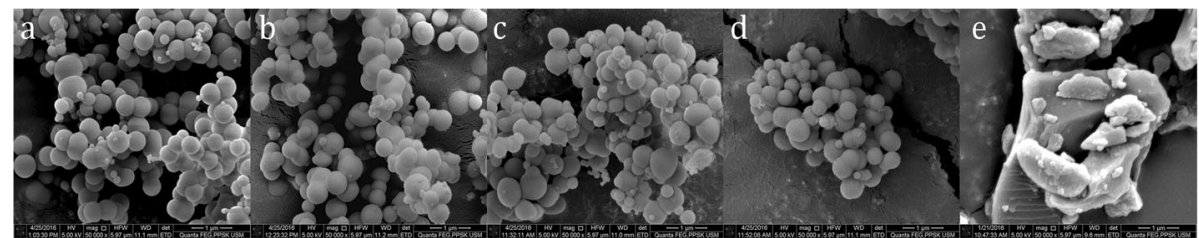

Fig. 5 SEM micrographs of a white, $\mathbf{b}$ yellow, $\mathbf{c}$ red, $\mathbf{d}$ pink SNP powders and e CFP

shows infrared (IR) spectra obtained from the samples which presented the typical absorption peak of silica. The peaks at 1095, 796 and $472 \mathrm{~cm}^{-1}$ correspond to the asymmetric and symmetric stretching as well as bending of the siloxane ( $\mathrm{Si}-\mathrm{O}-\mathrm{Si}$ ) bond. IR spectra of white and coloured SNP has showed minimal interspectra variances, indicating little or no change have taken place in the powder formulation after addition of corn starch and colour pigments.

\section{Application of fluorescent variant of SNP powder for fingerprint development}

The effectiveness of fingerprint developing powders can be evaluated from two aspects, namely clarity and contrast. Higher clarity can be achieved by selective particle adherence on the fingerprint ridge residue. In other words, fingerprints developed possess the highest similarities between the original ridge deposit and developed ridges. In addition, higher contrast is obtained by enhancing the fingerprint ridges and relatively diminishing the visibility substrate containing the fingerprint. An optimal fingerprint developing powder would produce developed fingerprint with high clarity and contrast. Figure 7 exhibits the fingerprints developed using fluorescent variants of the SNP powder and the CFP on multiple surfaces. CFP exhibited the highest contrast on most of the surfaces tested except for fluorescent paper, while yellow SNP powder possessed slightly lower fluorescence intensity. Red and pink SNP displayed poor contrast in comparison to CFP and yellow SNP powders. However, over powdering was observed in the fingerprints developed using the CFP. All powder performed poorly on the patterned leather surface owing to its uneven surface texture.

It was evident that CFP offered an enhanced contrast with the reduced clarity of fingerprint ridges as illustrated in Fig. 8a. This was thought due to the fact that commercial powder particles had inconsistent size and shapes. In comparison, the yellow SNP powder offered both good clarity and contrast of fingerprints (Fig. 8b)

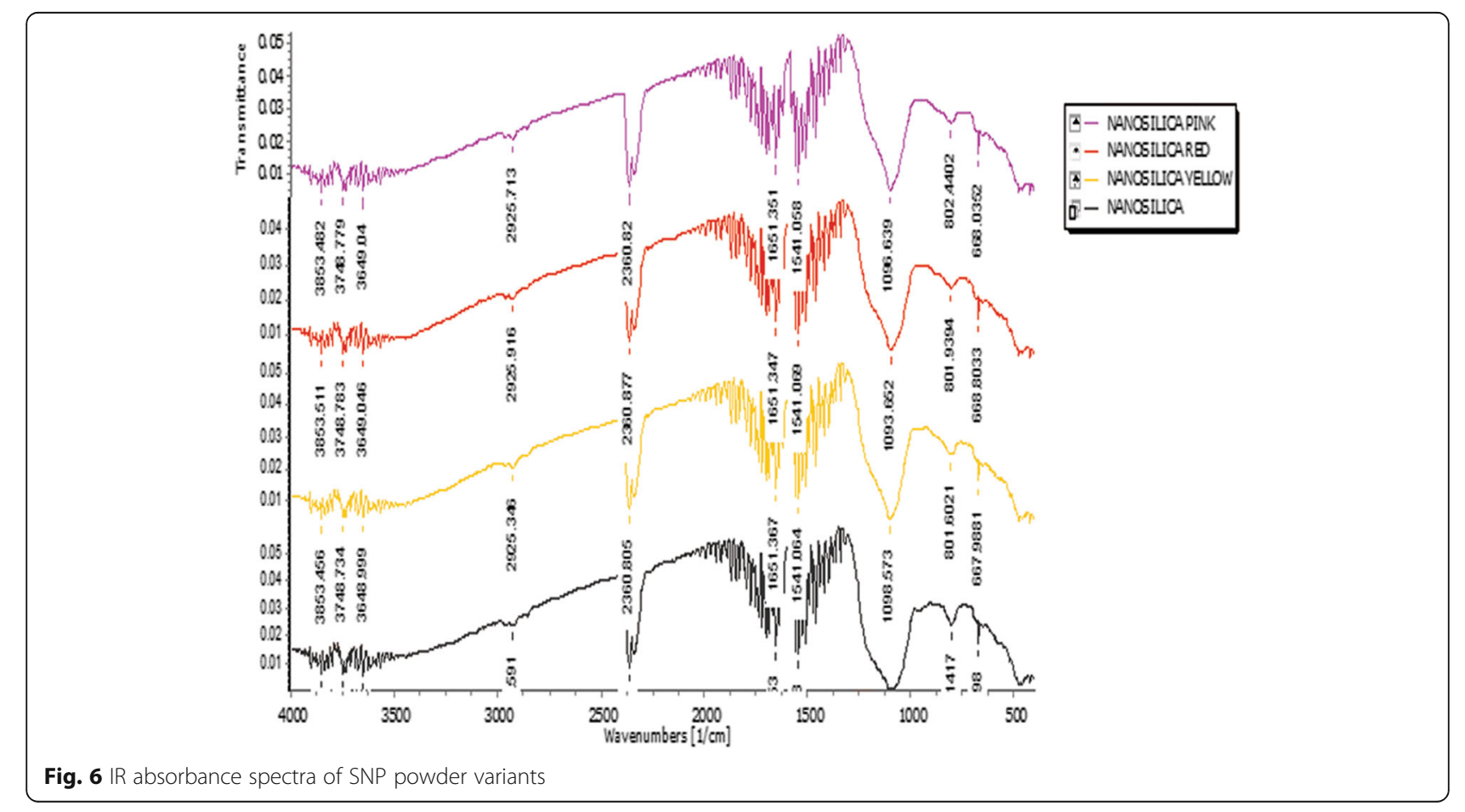



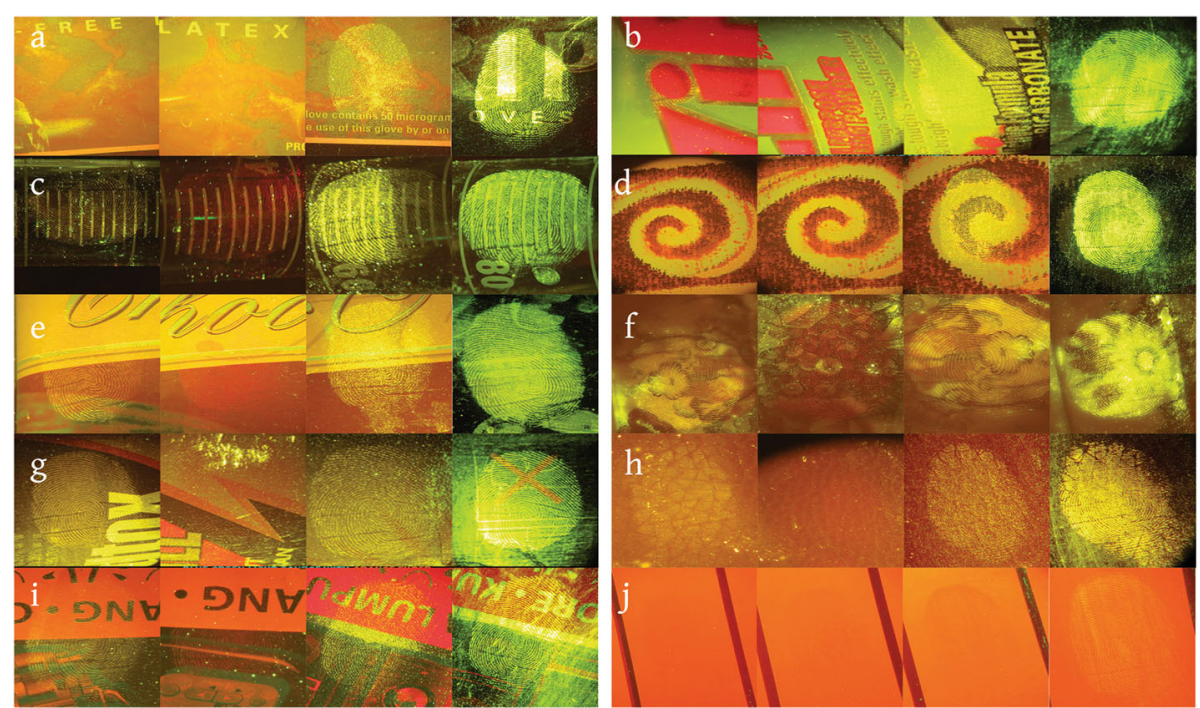

Fig. 7 Fingerprints developed using fluorescent variant of SNP powder and CFP on multiple surfaces a glossy paper box, $\mathbf{b}$ plastic bottle, $\mathbf{c}$ measuring cylinder, $\mathbf{d}$ wallpaper, e metal can, $\mathbf{f}$ porcelain cup, $\mathbf{g}$ metal can, $\mathbf{h}$ patterned leather, i plastic bag and $\mathbf{j}$ fluorescent paper

developed on non- and semi-porous surfaces in favour of the mono-dispersed spherical-shaped powder particles.

The toxicity of the powder is also another important aspect when considering new powder formulation for a regular application. Natural dye-doped SNP synthesised from rice husk would exhibit very minimal hazard for the users. The sizes of particles range in between 200 and $400 \mathrm{~nm}$ and thus, can be effectively filtered by the use of face masks. Comparatively, the commercial luminescent yellow powder contains Saturn yellow AX pigment and lycopodium. Material safety data sheet of this powder indicates that the powder is harmful upon prolonged exposure through inhalation and skin contact. This powder is also expensive as compared to regular black or white powders.

A considerable amount of literature has been published with regards to conferring luminescent property to fingerprint dusting powder. Various dyes and powders have been tested and proposed for such purpose and our study was proven to be concurrent with previous research (Liu et al. 2008). Despite primary particle size of $\sim 100$ to $500 \mathrm{~nm}$ was employed, very minimal

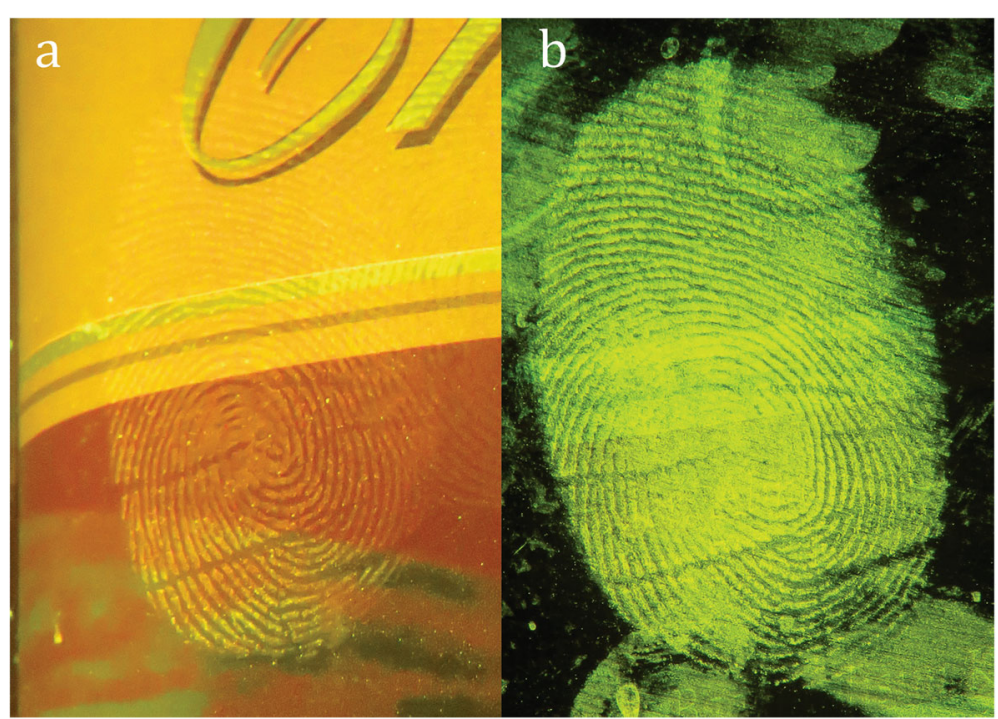

Fig. 8 Fingerprints developed using a yellow SNP powder and b CFP 
aggregation was observed throughout the powder particles' microscopic examination. This had increased the quality of the fingerprints developed.

It is noted that most of the researches focused on conferring luminescent property to the nanoparticles via synthetic dyes that are toxic in nature (Vujanovic et al. 2012). Many of the dyes cannot be released into the water circulation for fear that it may cause toxic effects and cannot be removed during water purification process (Gombert et al. 2017). The dyes used in this research were food dyes and natural extracts from plants albeit two synthetic dyes were also explored. However, only the yellow SNP powder dye doped using curcumin pigments extracted from turmeric was selected for fingerprint dusting application since it has exhibited the highest photoluminescence and stability. Similar findings were also reported by another study conducted to evaluate the efficacy of natural food colouring powder and festival colour powders for the development of fingerprints (Harish et al. 2011). In another instance, curcumin has also been applied as a wet particle suspension which produced similar shortcomings. The use of organic dye and SNP powder produced from $\mathrm{RH}$ overcomes both shortcomings of using a pure organic pigment powder because SNP alone was found to be highly specific to fingerprint ridges and it also retained the photoluminescence property of the curcumin dye. SNP particles act as a carrier for the dye molecules, simultaneously increasing the contrast through photoluminescence and providing good quality of the ridge details.

\section{Conclusion}

In summary, fluorescent variant of the spherical mono-dispersed SNP powder was successfully produced in this research by incorporating curcumin pigment extracted from turmeric. The fluorescent SNP powder exhibited a good contrast for fingerprint ridge enhancement without contributing to excess background fluorescence. Utilisation of spherical SNP synthesised from rice husk with luminescence conferred using natural pigment reduced the risk of toxicity to users. In addition, this powder has also enhanced the contrast and clarity of the developed fingerprint as effective as commercially available fluorescent powders.

\section{Abbreviations}

CFP: Commercial fluorescent powder; FALS: Forensic alternative light source: FESEM: Field emission scanning electron microscopy; FTIR: Fourier-transform infrared spectroscopy; IR: Infrared; KBr: Potassium bromide; SNP: Silica nanoparticle; UV: Ultraviolet; XRD: X-ray diffraction spectroscopy

\section{Acknowledgements}

Not applicable.

\section{Authors' contributions}

All authors contributed to the design of the study. RR performed the experiments. RR and NF analysed the data. All authors discussed and wrote the manuscript. All authors read and approved the final manuscript.

\section{Author's information}

Not applicable.

\section{Funding}

Financial support for this research by the Universiti Sains Malaysia (USM) Research Grant 1001/PPSK/812125.

\section{Availability of data and materials}

Please contact author for data requests.

\section{Ethics approval and consent to participate}

The human ethical clearance was approved by Human Research Ethics Committee, Universiti Sains Malaysia (reference number: USM/JEPeM/ 280.5(1.3)).

\section{Consent for publication}

Consent to publish was obtained from each participant.

\section{Competing interests}

The authors declare that they have no competing interests.

\section{Author details}

'Department of Diagnostics and Allied Health Sciences, Faculty of Health and Life Sciences, Management and Science University, 40100 Shah Alam, Selangor, Malaysia. ${ }^{2}$ Forensic Science Programme, School of Health Sciences, Universiti Sains Malaysia, 16150 Kubang Kerian, Kelantan, Malaysia.

${ }^{3}$ Biomedical Science Programme, School of Health Sciences, Universiti Sains Malaysia, 16150 Kubang Kerian, Kelantan, Malaysia.

Received: 18 July 2018 Accepted: 26 August 2019

Published online: 03 September 2019

\section{References}

Abu R, Yahya R, Neon S (2016) Production of high purity amorphous silica from rice husk. Procedia Chem 19:189-195

Adam F, Chew T-S, Andas J (2011) A simple template-free sol-gel synthesis of spherical nanosilica from agricultural biomass. I Sol-Gel Sci Technol 59:580-583

Altınöz S, Toptan S (2002) Determination of tartrazine and Ponceau-4R in various food samples by Vierordt's method and ratio spectra first-order derivative UV spectrophotometry. J Food Compos. Anal. 15:667-683

Azman AR, Mahat NA, Abdul Wahab R, Abdul Razak Fl, Hamzah HH (2018) Novel safranin-tinted Candida rugosa lipase nanoconjugates reagent for visualizing latent fingerprints on stainless steel knives immersed in a natural outdoor pond. Int J Mol Sci. 19(6):1576

Balaji G, Rekha RK, Ramalingam A (2011) Nonlinear characterization of safranin O dye for application in optical limiting. Acta Phys Pol A 119:359-363

Becue A, Champod C, Margot P (2007) Use of gold nanoparticles as molecular intermediates for the detection of fingermarks. Forensic Sci Int 168:169-176

Becue A, Moret S, Champod C, Margot P (2009) Use of quantum dots in aqueous solution to detect blood fingermarks on non-porous surfaces. Forensic Sci Int 191:36-41

Becue A, Scoundrianos A, Champod C, Margot P (2008) Fingermark detection based on the in situ growth of luminescent nanoparticles-towards a new generation of multimetal deposition. Forensic Sci Int 179:39-43

Bergeron J (2003) Development of bloody prints on dark surfaces with titanium dioxide and methanol. J Forensic Identif 53:149-161

Bharat LK, Raju GSR, Yu JS (2017) Red and green colors emitting spherical-shaped calcium molybdate nanophosphors for enhanced latent fingerprint detection. Sci Rep 7:1-14

Bogner C, Schmiedinger I, Huwe B (2011) Rapid estimation of brilliant blue concentrations in soil via diffuse reflectance spectroscopy. Geoderma 164:95-98

Carmona VB, Oliveira RM, Silva WTL et al (2013) Nanosilica from rice husk: Extraction and characterization. Ind Crop Prod 43:291-296

Chen D, Wu Z (2009) Study on extraction and purification process of Capsicum red pigment. J Agric Sci 1:94-100

Chen H, Wang W, Martin JC et al (2013) Extraction of lignocellulose and synthesis of porous silica nanoparticles from rice husks: a comprehensive utilization of rice husk biomass. ACS Sustain Chem Eng 1:254-259 
Cheriaa J, Khaireddine M, Rouabhia M, Bakhrouf A (2012) Removal of triphenylmethane dyes by bacterial consortium. Sci World J 2012:1-9

Choi MJ, McBean KE, Ng PHR et al (2008) An evaluation of nanostructured zinc oxide as a fluorescent powder for fingerprint detection. J Mater Sci 43:732-737

Choi MJ, Smoother T, Martin AA et al (2007) Fluorescent TiO2 powders prepared using a new perylene diimide dye: applications in latent fingermark detection. Forensic Sci Int 173:154-160

Dorbut T, Benchawattananon R (2016) Small particle reagent based on natural dyes for developing latent fingerprints on non-porous wet surfaces. In: The 2016 Management and Innovation Technology International Conference (MITiCON-2016) Small, pp 225-228

Errington B, Lawson G, Lewis SW, Smith GD (2016) Micronised Egyptian blue pigment: a novel near-infrared luminescent fingerprint dusting powder. Dye Pigment 132:310-315

Garg RK, Kumari H, Kaur R (2011) A new technique for visualization of latent fingerprints on various surfaces using powder from turmeric: a rhizomatous herbaceous plant (Curcuma longa). Egypt J Forensic Sci 1:53-57

Gaskell C, Bleay SM, Ramadani J (2013) Natural yellow 3: a novel fluorescent reagent for use on grease-contaminated fingermarks on nonporous dark surfaces. J Food Sci Technol 63:274-285

Ghorbani F, Sanati AM, Maleki M (2015) Production of silica nanoparticles from rice husk as agricultural waste by environmental friendly technique. Environ Stud Persian Gulf 2:56-65

Gombert P, Biaudet H, de Seze R et al (2017) Toxicity of fluorescent tracers and their degradation byproducts. Int J Speleol 46:23-31

Guzman M, Flores B, Malet L, Godet S (2018) Synthesis and characterization of zinc oxide nanoparticles for application in the detection of fingerprints. Mater Sci Forum 916:232-236

Harish K, Ramanjit K, Rakesh KG (2011) New visualizing agents for latent fingerprints: Synthetic food and festival colors. Egypt J Forensic Sci 1:133-139

Hessien MM, Rashad MM, Zaky RR et al (2009) Controlling the synthesis conditions for silica nanosphere from semi-burned rice straw. Mater Sci Eng B 162:14-21

International Fingerprint Research Group (IFRG) (2014) Guidelines for the assessment of fingermark detection techniques. J Forensic Ident. 64:174-200

Jasuja OP, Kumar P, Singh G (2015) Development of latent fingermarks on surfaces submerged in water: optimization studies for phase transfer catalyst (PTC) based reagents. Sci Justice 55:335-342

Kim Y, Jung H, Lim J et al (2016) Rapid imaging of latent fingerprints using biocompatible fluorescent silica nanoparticles. Langmuir 32:8077-8083

Lee HC, Gaensslen RE (2012) Lee and Gaensslen's: advances in fingerprint technology, Third Edit. CRC press, Boca Raton

Lennard C (2014) Fingermark detection and identification : current research efforts. Aust J Forensic Sci 46:293-303

Li D, Zhu X (2011) Short-period synthesis of high specific surface area silica from rice husk char. Mater Lett 65:1528-1530

Li Z, Wang Q, Wang Y et al (2018) Background-free latent fingerprint imaging based on nanocrystals with long-lived luminescence and $\mathrm{pH}$-guided recognition. Nano Res 11:6167-6176

Liu L, Gill SK, Gao Y et al (2008) Exploration of the use of novel SiO2 nanocomposites doped with fluorescent Eu3+/sensitizer complex for latent fingerprint detection. Forensic Sci Int 176:163-172

Low WZ, Khoo BE, Aziz ZBA et al (2015) Application of acid-modified Imperata cylindrica powder for latent fingerprint development. Sci Justice 55:347-354

Lu P, Hsieh YL (2012) Highly pure amorphous silica nano-disks from rice straw. Powder Technol 225:149-155

Mor S, Manchanda CK, Kansal SK, Ravindra K (2017) Nanosilica extraction from processed agricultural residue using green technology. J Clean Prod 143: 1284-1290

Moret S, Bécue A, Champod C (2014) Nanoparticles for fingermark detection: an insight into the reaction mechanism. Nanotechnology 25:1-10

Narayana B, Divya NS (2010) A new method for spectrophotometric determination of colchicoside. J Sci Ind Res (India) 69:368-372

Newland TG, Moret S, Bécue A, Lewis SW (2016) Further investigations into the single metal deposition ( SMD II) technique for the detection of latent fi ngermarks. Forensic Sci Int 268:62-72

Noushad M, Ab Rahman I, Che Zulkifli NS et al (2014) Low surface area nanosilica from an agricultural biomass for fabrication of dental nanocomposites. Ceram Int 40:4163-4171

Patel P, Patel L, Patel D (2012) A color cosmetic composition with increased color intensity
Prabakaran E, Pillay K (2019) Synthesis and characterization of fluorescent N-CDs/ ZnONPs nanocomposite for latent fingerprint detection by using powder brushing method. Arab J Chem

Rajan R, Zakaria Y, Shamsuddin S, Nik Hassan NF (2016) Synthesis and characterisation of silica nanoparticles from rice husk for latent fingermark development. In: 21st National Conference on Medical and Health Science, Kubang Kerian, p 31

Singh K, Sharma S, Garg RK (2013) Visualization of latent fingerprints using silica gel G : a new technique. Egypt J Forensic Sci 3:20-25

SIRCHIE (2012) LL7052D Yellowescent fluorescent latent print powder, Youngsville

Sodhi GS, Kaur J (2008) A novel, nanoparticle-size fingerprint dusting composition based on Eosin B stain. Indian Police J LV, pp 46-49

Sodhi GS, Kaur J (2017) Multimetal deposition method for detection of latent fingerprints: a review. Egypt J Forensic Sci 7:1-7

Vaibhav V, Vijayalakshmi U, Roopan SM, Mohana Roopan S (2015) Agricultural waste as a source for the production of silica nanoparticles. Spectrochim Acta A Mol Biomol Spectrosc 139:515-520

Vujanovic S, Kheng Y, Vladimir G (2012) Natural fruit extracts as non-toxic fluorescent dyes for staining fungal chlamydospores. World J Microbiol Biotechnol 28:387-390

Wang J, Ma Q, Liu H et al (2017) Time-Gated imaging of latent fingerprints and specific visualization of protein secretions via molecular recognition timegated imaging of latent fingerprints and specific visualization of protein secretions via molecular recognition. Anal Chem:1-8

Wang W, Martin JC, Fan X et al (2012) Silica nanoparticles and frameworks from rice husk biomass. ACS Appl Mater Interfaces 4:977-981

Zhao D, Ma W, Xiao X (2018) The Recognition of sweat latent fingerprints with green-emitting carbon dots. Nanomaterials 8:1-10

\section{Publisher's Note}

Springer Nature remains neutral with regard to jurisdictional claims in published maps and institutional affiliations.

\section{Submit your manuscript to a SpringerOpen ${ }^{\circ}$ journal and benefit from:}

- Convenient online submission

- Rigorous peer review

- Open access: articles freely available online

- High visibility within the field

- Retaining the copyright to your article

Submit your next manuscript at $\boldsymbol{\nabla}$ springeropen.com 